\title{
VARIABILITY OF SOLAR ERUPTIONS DURING CYCLE 23
}

\author{
N. Gopalswamy ${ }^{1}$, S. Nunes ${ }^{2}$, S. Yashiro ${ }^{2}$, And R. A. Howard ${ }^{3}$ \\ ${ }^{1}$ NASA Goddard Space Flight Center, Greenbelt, MD 20771, USA \\ ${ }^{2}$ The Catholic University of America, Washington DC 20064, USA \\ ${ }^{3}$ Naval Research Laboratory, Washington DC 20064, USA
}

\begin{abstract}
We report on the solar cycle variation of the rate of coronal mass ejections (CMEs), their mean and median speeds, and the rate of type II radio bursts. We found that both CME rate and speed (mean and median) increased from solar minimum to maximum by factors of 10 and 2, respectively. The CME rate during solar maximum is nearly twice the rates quoted previously. Large spikes in the speed variation were due to active regions that were highly active. The poor correlation between metric and DH type II bursts is confirmed, and the difference is attributed to the different Alfven speeds in the respective source regions.
\end{abstract}

\section{INTRODUCTION}

The sunspot cycle is an important form of solar variability that indicates the extent of closed magnetic field structures on the Sun and hence important to study the origin of coronal mass ejections (CMEs). Webb and Howard (1994) established that CME occurrence rate tracks the solar activity cycle in both amplitude and phase. Furthermore, they found that CMEs accounted for a significant fraction of the solar wind mass flux near solar maximum. There is another important aspect of CME rates especially when they have high speeds: CMEs also drive shocks, which in turn accelerate electrons (detected in situ and inferred from type II radio bursts) and ions (detected in situ). Thus radio bursts and solar energetic particles (SEPs) depend on subsets of CMEs. In this paper, we report the rates of CMEs, metric type II bursts, Decameter-Hectometric (DH) Type II bursts, and those of special populations of CMEs such as fast and wide (FWFW) CMEs and SEP-associated CMEs for the period 1996-2001.

\section{DATA}

The Solar and Heliospheric Observatory (SOHO) mission's Large Angle and Spectrometric Coronagraph (LASCO) routinely records CMEs. More than 5000 CMEs detected during 1996-2001 by LASCO, which have been measured and cataloged on line (http://cdaw.gsfc.nasa.gov/CME list). The catalog contains all the CMEs manually detected. When it is possible to make at least two height-time measurements for a CME, we obtained its speed. The primary attributes of a CME listed in the catalog are: (1) linear speed, (2) central position angle, and (3) angular width. All these measurements were made in the sky plane and no attempt has been made to correct for projection effects. Since CMEs have been known to accelerate, decelerate or have constant speed in the coronagraph field of view (Gopalswamy et al., 2001), the catalog also lists speeds obtained from quadratic fit to the height-time measurements at the last measurement point and at 20 Rs. In this paper, we use the speed obtained from linear fit to better compare to previous LASCO studies. We use these measurements to study the solar cycle variation of CME properties. We collected metric type II bursts primarily from the Solar Geophysical Data (SGD) with supplements from the web sites of individual observatories such as Potsdam, IZMIRAN, and the Hiraiso Solar Terrestrial Research Center. Type II bursts in the IP medium were obtained by the Radio and Plasma Waves Experiment on board the Wind spacecraft (Bougeret et al., 1995). These bursts are also cataloged on line (http://www-lep.gsfc.nasa.gov/waves). The solar energetic particle (SEP) events were collected from the NOAA web site. We considered only events with intensity $>10$ particles $/\left(\mathrm{s} \mathrm{cm}^{2} . \mathrm{sr}\right)$ in the $>10 \mathrm{MeV}$ channel. Properties 
of the associated CMEs, flares and radio bursts are assembled in the Living with a Star (LWS) coordinated data analysis workshop (CDAW) web site (http://cdaw.gsfc.nasa.gov/LWS).

\section{CME RATE}

The occurrence rate of CMEs is determined as an average value for each Carrington Rotation (CR), similar to Cliver et al. (1994) and St. Cyr et al. (2000). This value $\left(\mathrm{R}_{\mathrm{obs}}\right)$ is obtained by counting all the CMEs reported in a CR and dividing the sum by the LASCO operational time $\left(\mathrm{T}_{\mathrm{u}}\right)$ over that CR. This of course, is not the true rate because CMEs might have occurred during LASCO downtimes. To get an estimate of the error bar, we obtained the following from LASCO operator logs and movies for each CR: total operational time, total down time $\left(T_{d}\right)$, and maximum number of CMEs $\left(R_{\max }\right)$ observed per day. These determine the upper and lower error bars to the CME rate. $T_{d}$ was obtained by accumulating all data gaps greater than $\sim 3 \mathrm{hr}$ (see St. Cyr et al., 2000 for details). The lower limit to the CME rate is obtained by assuming that no other CMEs occurred during the CR than $\mathrm{T}_{\mathrm{u}} \mathrm{R}_{\mathrm{obs}}$. Thus, the lower limit is given by $\mathrm{R}_{\text {obs }} / 27.34$, where we have taken the length of the $\mathrm{CR}$ to be 27.34 days. The upper limit to the CME rate is obtained by assuming that, during $T_{d}$, CMEs occurred at a rate given by $R_{\max }$ during that CR. This gives an additional $T_{d} R_{\max }$ CMEs to be added to the observed number of CMEs. The total $\left(T_{u} R_{o b s}+T_{d} R_{\text {max }}\right)$ is then divided by the length of the CR (27.34 days) to get the upper limit. Thus the actual CME rate is somewhere between $R_{\text {obs }} / 27.34$ and $\left(T_{u} R_{o b s}+T_{d} R_{\text {max }}\right) / 27.34$. We must point out that some narrow CMEs form close to the disk center might have been missed by LASCO by virtue of the occultation technique used by coronagraphs. This will push the upper limit even higher, but as St. Cyr et al. (2000) argued, the fraction of such CMEs may be negligible. Figure 1 shows the CME daily rate as a function of CR.

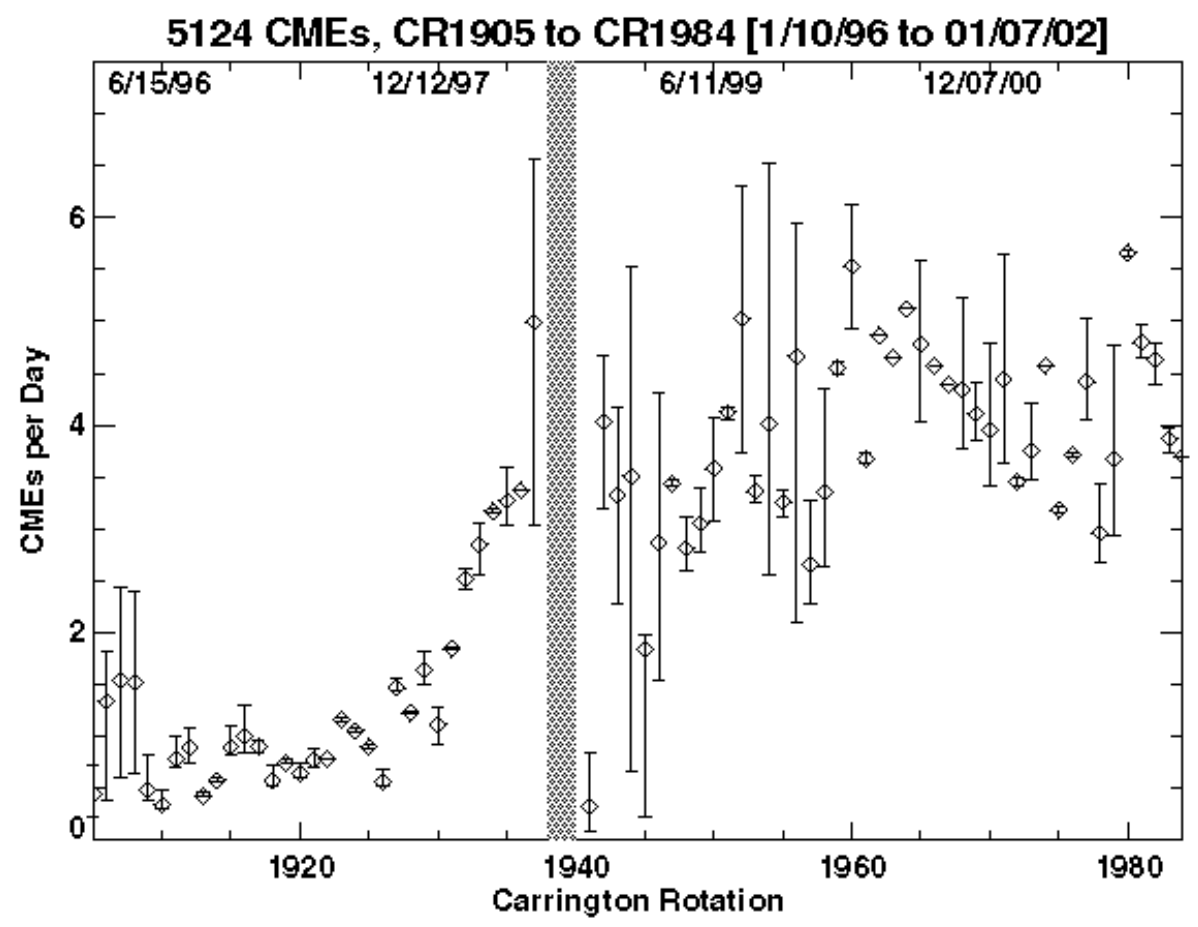

Fig. $1 \mathrm{CME}$ daily rate as a function of Carrington Rotation (CR). The diamonds represent the observed rate. The error bars are computed based on the total uptime, downtime and maximum observed rate in each CR. See text for explanation. The vertical shaded region represents an extended down time when SOHO was disabled from 24 June 1998 to 15 October 1998.

The rate up to CR 1937 (1998 June) is roughly the same as in St. Cyr et al. (2000) with minor changes due to additional CMEs identified later on. The rate was roughly flat until about CR 1925 at a value of 0.5 per day. After that the rate increased rapidly to reach a value of 5.05 within a year. The low 
rate of 0.46 during CR 1941 is due to the fact that most of this CR period fell in the SOHO mission interruption period except for the last seven days. Other than that the rate hovered around 4.5 until the end of the study period. There were 5 rotations with an occurrence rate exceeding 5 , including the first peak during CR 1937. The largest rate (5.66) occurred during CR 1980 (2001 August) with a very small error bar. The lowest rate occurred during CR 1910 (June 1996), right at the solar minimum. Thus the CME rate increased by a factor of 18 between the CRs 1910 and 1980, representing an order of magnitude increase during the solar maximum compared to the solar minimum. In an earlier analysis of the LASCO CMEs during the minimum and rising phase of the solar cycle, St. Cyr et al. (2000) found that the CME rate agreed in amplitude and phase with that obtained by Webb and Howard (1994) from Skylab, SMM, Helios, and Solwind observations. Webb and Howard (1994) found a rate of 0.31 to 0.77 CMEs/day for the solar minimum years and 1.75 to $3.11 \mathrm{CMEs} /$ day for the solar maximum years. For the minimum period, our rate agrees with that of Webb and Howard (1994), but for the maximum period we get a rate almost twice that of Webb and Howard (1994) or Cliver (1994). Note that we have not corrected for visibility function, whereas Webb and Howard had corrected for both visibility function and duty cycle. It is not clear if this discrepancy is peculiar to this solar cycle or it can be attributed to the unprecedented sensitivity of the LASCO coronagrpah. The CME mass flux may also have to be revised according to the higher rate.
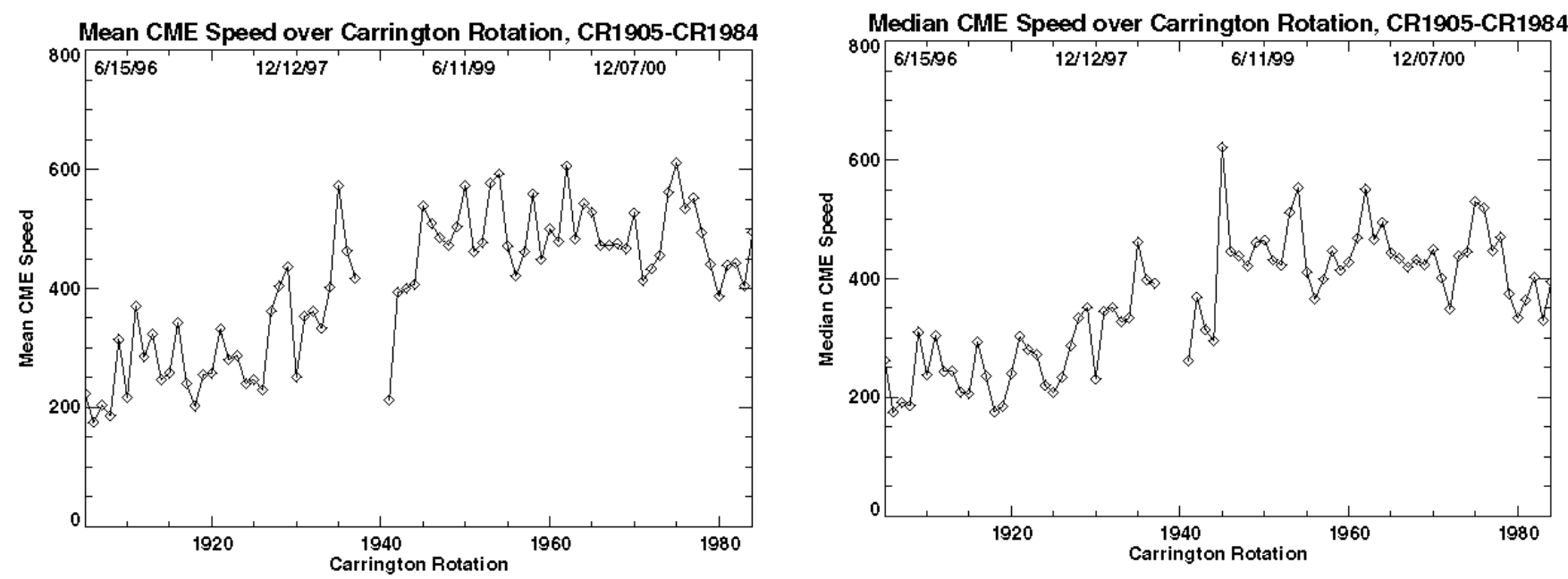

Fig. 2. (left) Mean speed and (right) median speed of CMEs as a function of Carrington Rotation number. The gap corresponds to the SOHO mission interruption during June to September 1998.

\section{CME SPEED}

We computed the mean and median speeds of the observed CMEs in each CR and plotted them as a function of CR in Figure 2. Near solar minimum, the mean speed is around $275 \mathrm{~km} / \mathrm{s}$ and increases to about $550 \mathrm{~km} / \mathrm{s}$ during solar maximum. Thus the mean speed increases from solar minimum to maximum to max by a factor of $\sim 2$. The speed variation appears to be somewhat smoother than CME rate, especially during solar maximum. The large peaks in the mean speed plot come from some super active regions that produced frequent high speed CMEs. The median speed in Fig. 2 has a similar tendency, but has less number of spikes. Both mean and median speeds show a step-like behavior between CRs 1930 and 1940, somewhat similar to that of the CME rate. The large spike in CR 1929 is due to AR 8100, which produced a large number of fast CMEs, resulting in a mean speed of $437 \mathrm{~km} / \mathrm{s}$. The second spike in CR 1935 with a mean speed of $573 \mathrm{~km} / \mathrm{s}$ was mostly due to fast CMEs from AR 8210. The largest peak in the mean speed is again due to contributions from AR 9236 during CR 1970 (November 2000). The largest spike (CR 1945) in the median speed is an artifact because of a SOHO data gap from 1998 Dec 20 to 1999 Feb 5, which included most of CR 1945 (January 12 to February 8, 1999). There were only 6 CMEs during the uptime of 3 days (February 5-8, 1999) and most of them were faster than $600 \mathrm{~km} / \mathrm{s}$, and hence resulted in a large median speed. In semiannual rates, these peaks smooth out, clearly revealing the increase in mean and medium speeds 
from minimum to maximum. The speed data from the Solwind coronagraph indicated increase in the mean speed towards solar maximum (St. Cyr et al., 1999). However, Hundhausen (1999) did not find a clear trend in SMM CME speeds. The highest speed $(458 \mathrm{~km} / \mathrm{s})$ during the SMM era was found during solar minimum (1985). There is some discrepancy between SMM and P78-1 data during this period, which needs further investigation. Ivanov and Obridko (2001) found a peak near solar minimum in the semiannual averages of speeds from SMM and Solwind data, in addition to peaks in the maximum epochs (years 1981-1982 and 1989). The SOHO data presented above have shown beyond doubt that on the average CMEs are faster during solar maximum.

\section{SPECIAL POPULATIONS}

CMEs occurring close to the disk center are likely to propagate towards Earth and cause large geomagnetic storms, provided the CME magnetic field has a southward component. These are generally halo CMEs (Howard et al., 1982) because they expand rapidly to appear to surround the occulting disk within the first couple of hours of the onset. CMEs originating far from the central meridian may be less effective in producing geomagnetic storms because the CME does not reach the earth. However, if these limb CMEs drive shocks, then the shock sheaths may arrive at Earth with a southward magnetic field component and cause geomagnetic storms. Halo CMEs need not be fast, but shockdriving CMEs are fast. It has been established that fast and wide CMEs are closely associated with interplanetary type II radio bursts (Gopalswamy et al., 2001b) in the decameter-hectometric (DH) wavelengths and large SEP events (Gopalswamy et al. 2002). Figure 3 shows the annual distributions of various CME populations from 1996 to 2001 . Note that the halo CME population ( $2^{\text {nd }}$ panel) is only a small fraction of all the CMEs. Even though there were 1536 CMEs in year 2000, there were only 120 halo CMEs $(<10 \%)$. On the other hand, the fraction of fast $(>900 \mathrm{~km} / \mathrm{s})$ and wide (width $>60 \mathrm{deg})$ CMEs $\left(3^{\text {rd }}\right.$ panel) is still smaller $(\sim 5 \%)$. If we further restrict the source longitudes to western events $\left(4^{\text {th }}\right.$ panel), we get only 42 events $(<3 \%)$. Most of these fast and wide western events were associated with SEP events. In the bottom panel, we have shown the distribution of CMEs that produce DH type II bursts (DH CMEs). Note that the number of DH CMEs is very similar to that of the fast and wide CMEs, as one would expect. Thus for space weather applications, we need to consider two populations: the frontside halos and the fast and wide western hemispheric events. Of course, there are occasional eastern events that produce large SEP events. The number of DH CMEs is twice that of the fast and wide western events because radio emission does not depend on the connectivity.
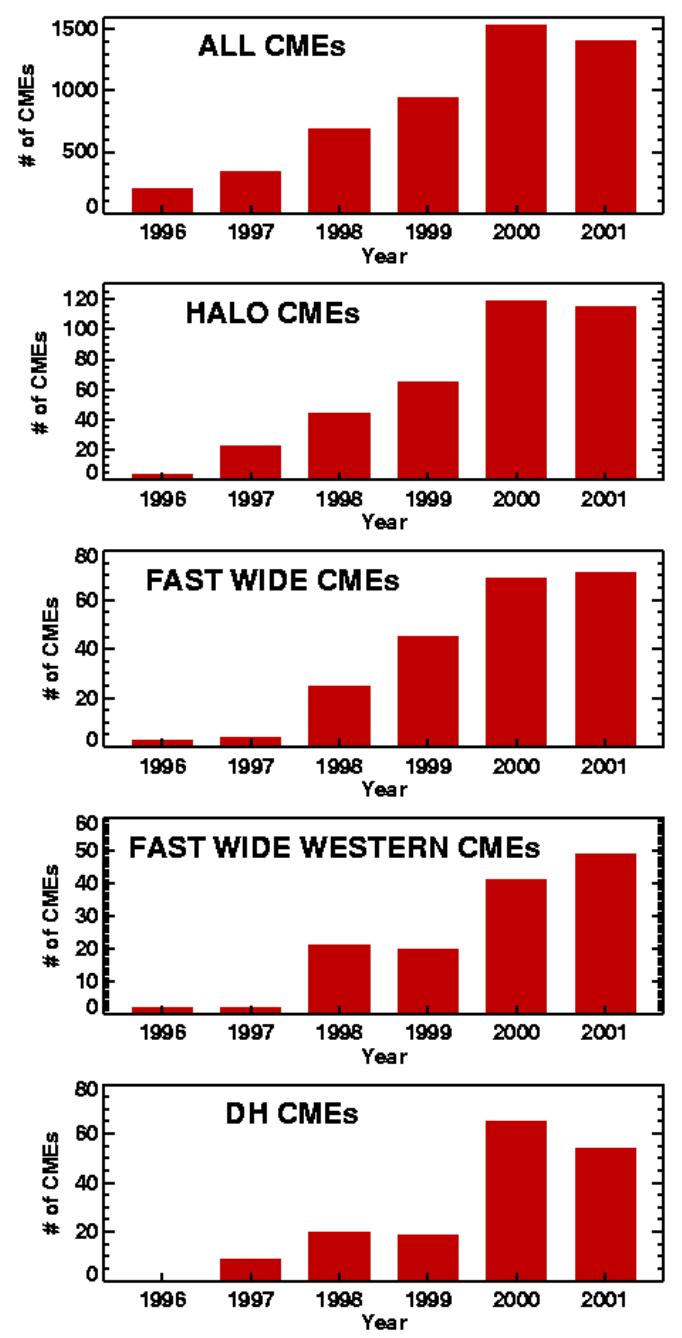

Fig.3. Annual distributions of CMEs

\section{TYPE II RADIO BURSTS}

Type II radio bursts occur in metric to kilometric wavelengths, corresponding to a few hundred $\mathrm{MHz}$ to about $30 \mathrm{kHz}$ or even lower. This frequency range corresponds to the entire Sun-earth connected space because the frequency of radio emission is closely related to the local plasma density in the corona and IP medium. It is known for a long time that kilometric type II bursts are due to CME-driven shocks, and it has recently been shown to be true for DH type II bursts (Gopalswamy et al., 2001b). The source of metric type II bursts, on the other hand, has been controversial, because a large number of super-Alfvenic disturbances are possible in the vicinity of the eruptions (Gopalswamy et al., 1998; Cliver et al., 1999). Gopalswamy et al. (1998) suggested that the driver of coronal shocks responsible for metric type II bursts must be short-lived because most of them are not seen at 
lower frequencies corresponding to the IP medium. This was also pointed out to be the reason for a poor correlation between coronal and DH type IIs. Recently, Gopalswamy et al. (2001a) invoked the Alfven speed peak (Krogulec et al., 1994; Mann et al., 1999) around 3 Rs in the corona to explain the poor correlation between metric and DH type II bursts. The DH domain starts roughly around the Alfven speed peak. It must be easier for CMEs to drive shocks on either side of this peak because of the low Alfven speeds prevailing in these regions. This also provides a natural explanation as to why a large number of metric type II bursts are associated with slow CMEs. More recently, Lara et al. (2003) looked at metric type II bursts without associated DH type II bursts and found that they were associated with CMEs faster and wider than the general population of CMEs. On the other hand CMEs associated with metric type II bursts were slower and narrower than DH CMEs. Thus the energy of CMEs is progressively higher to be associated with metric and DH type II bursts. Thus, it seems to be a reasonable hypothesis to consider that metric type II bursts are also closely associated with CMEs.

Figure 4 compares the solar cycle variation of type II burst rates binned by CRs. The total number of DH type II bursts is only 220 during the study period (1996-2001), while there were 606 metric type II bursts. That is, the number of metric type II bursts is three times that of DH type II bursts. Lack of correlation between the metric and DH type II numbers is evident if we compare CR by CR. For example, there was no DH type II burst in CR 1943, but there was a dozen metric type II bursts. The largest number (27) of metric type II bursts occurred in CR 1980 , but there were only 8 DH type II bursts. There were 154 CMEs in that CR, but the mean speed of CMEs
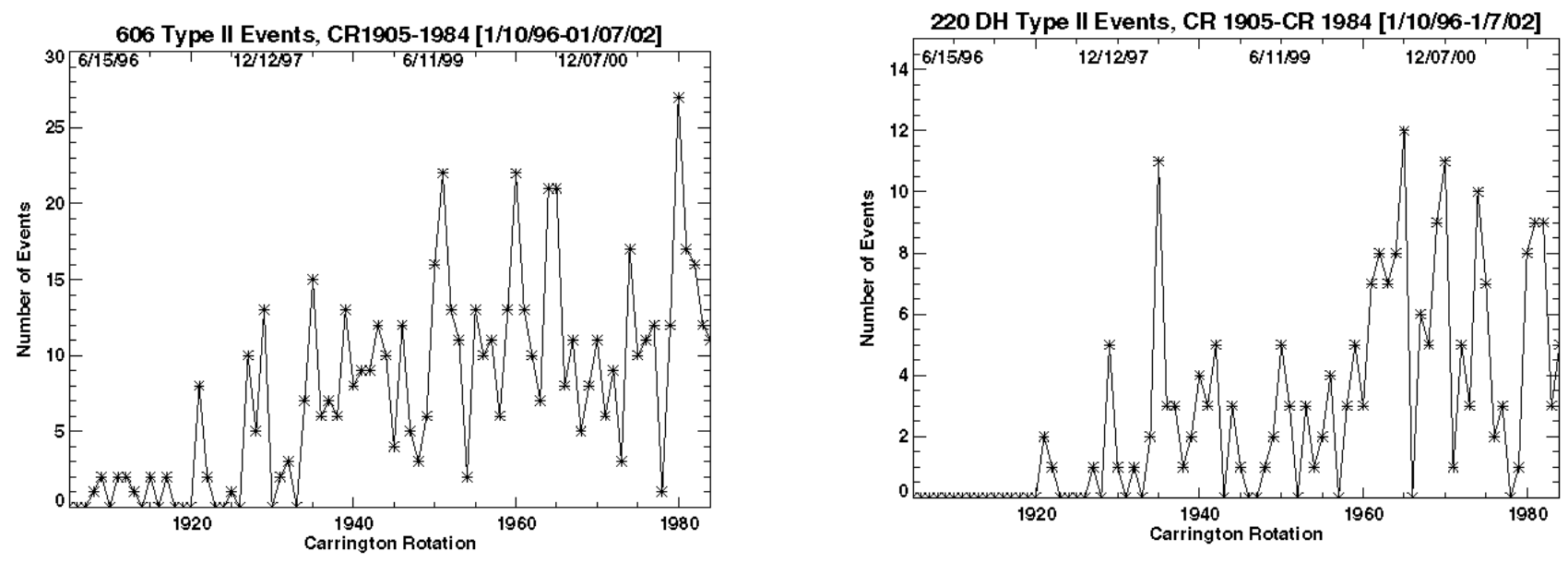

Fig. 4 Number of metric (left) and DH (right) type II bursts per Carrington rotation.

was only $384 \mathrm{~km} / \mathrm{s}$, which explains why there was a paucity of DH type II bursts. On the other hand, if we consider the largest DH peak early in the cycle (CR 1935), there were roughly the same number of metric (15) and DH (11) type II bursts. The number of CMEs was relatively small (83), but the mean speed during CR 1935 was much larger $(573 \mathrm{~km} / \mathrm{s}$, see Fig. 2) than in CR 1980. It is clear that the CME speed plays an important role for DH type II bursts. Gopalswamy et al. (2003) showed that the DH type II rate tracks the rate of fast and wide CMEs, which is also clear from Fig. 3. Metric type II bursts seem to require a lower threshold speed, but the properties of the ambient medium (density, magnetic field, and temperature that decide the characteristic speeds) are also important. Therefore, we need to consider not only the energy of the CMEs, but also the nature of the medium into which they are launched to understand the shock-driving capability of the CMEs.

\section{SUMMARY}

We studied the solar cycle variation of CME daily rate, mean and median speeds of CMEs, and the rate of type II radio bursts in the metric and $\mathrm{DH}$ domains. We find that (1) there is an order of magnitude increase in CME rate from the solar minimum (0.5/day) to maximum ( $>5 /$ day), (2) the maximum rate is significantly higher than previous estimates, (3) the mean and median speeds of CMEs also increase from minimum to maximum, but by a smaller factor of 2, (4) number of metric type II bursts (summed over CR) tracks CME rate, but the CME speed seems to be only of secondary importance, and (5) for DH type II bursts the CME speed is important. We also studied the annual distributions of a number of special populations of CMEs (halo CMEs, fast and wide CMEs, fast and wide western hemispheric CMEs, and DH CMEs). We found that only a small fraction of all the CMEs are important for space weather purposes: these are the front-side halo CMEs that 
originate from close to the central meridian (for geomagnetic storms) and the fast and wide CMEs from the western hemisphere (for large SEP events). Since DH type II bursts are associated with fast and wide CMEs, they may be good indicators of SEP-producing CMEs if we know that the bursts originate from the western hemisphere.

\section{ACKNOWLEDGMENTS}

We thank all the members of the LASCO Consortium (Naval Research Laboratory, USA; University of Birmingham, UK; Laboratoire d'Astronomie Spatiale, France; and Max Planck Institut fur Aeronomie, Germany) who built the coronagraphs and acquired the data used in this work. The preliminary list of CMEs generated by $\mathrm{O}$. C. St Cyr, S. P. Plunkett, and G. Lawrence have been very useful for further measurements. N. Rich formatted the LASCO data suitable for height-time measurements by G. Michalek and A. Rosas. We thank J.-L. Bougeret and M. L. Kaiser for making Wind/WAVES data available online. SOHO is a project of international collaboration between ESA and NASA. This work was supported by United States NASA/LWS, Air Force Office of Scientific Research, and NSF/SHINE (ATM02045880).

\section{REFERENCES}

Bougeret, J.-L., M. L. Kaiser, P. J. Kellog et al., Waves: The Radio and Plasma Wave Investigation on the Wind Spacecraft, Space Sci.Rev., 71, 231-263, 1995.

Cliver, E. W., O. C. St. Cyr, R. A. Howard, and P. S. McIntosh, Rotation averaged rates of coronal mass ejections and dynamics of polar crown filaments, in Solar coronal Structures, IAU Colloquium 144, eds. V. Rusin, P. Heinzel, and J.-C. Vial, VEDA Publishing Company, Bratislava, p.83-89, 1994.

Cliver, E. W., D. F. Webb, and R A. Howard, On the origin of solar metric type II bursts, Solar Phys., 187, 89114,1999

Gopalswamy, N., et al., Origin of coronal and interplanetary shocks - A new look with WIND spacecraft data, $J$. Geophys. Res., 103, 307-316, 1998

Gopalswamy, N., A. Lara, M. L. Kaiser and J.-L. Bougeret, Near-Sun and near-Earth manifestations of solar eruptions, , J . Geophys. Res., 105, 25261-25278, $2001 \mathrm{a}$.

Gopalswamy, N., S. Yashiro, M L. Kaiser, R. A. Howard and J.-L. Bougeret, Characteristics of coronal mass ejections associated with long-wavelength type II radio bursts, J. Geophys. Res., 106, 29219-29230, 2001 b.

Gopalswamy, N., S. Yashiro, G. Michalek et al., Interacting coronal mass ejections and solar energetic particles, Astrophys. J., 572, L103-L107, 2002.

Gopalswamy, N. et al., Large solar energetic particle events of cycle 23: a global view, Geophys. Res. Lett., 30, SEP3-1, 2003.

Ivanov, E. V. and V. N. Obridko, Cyclic variation of CME velocity, Solar Phys., 198, 179-195, 2001.

Krogulec, M., Z. E. Musielak, S. T. Suess, S. F. Nerney, and R. L. Moore, The Role of Alfven Waves in Solar Wind Acceleration, J. Geophys. Res., 99, 23489-23501, 1994.

Lara, A. N. Gopalswamy, S. Nunes, G. Munoz., and S. Yashiro, A statistical study of CMEs associated with metric type II bursts, Geophys. Res. Lett., 30, SEP4-1, 2003.

Mann, G., A. Klassen, C. Estel, and B. J. Thompson, Coronal Transient Waves and Coronal Shock Waves, in ESA Special Publications 446, eds. J.-C. Vial and B. Kaldeich-Schümann., p.477-481, 1999.

Howard, R. A., D. J. Michels, N. R. Sheeley, and M. J. Koomen, The observation of a coronal transient directed at earth, Astrophys. J., 263, L101-L104, 1982.

St Cyr, O. C., J. . Burkepile, A. J. Hundhausen, and A. R. Lecinski, A comparison of ground-based and spacecraft observations of coronal mass ejections from 1980-1989, J. Geophys. Res., 104, 12493-12506, 1999.

St Cyr, O. C. et al., Properties of coronal mass ejections: SOHO LASCO observations from January 1996 to June 1998, J. Geophys. Res., 105, 18169-18186, 2000.

Webb, D. F. and R. A. Howard, The solar cycle variation of coronal mass ejections and the solar wind mass flux, J. Geophys. Res., 99, 4201-4220, 1994. 